\title{
Technological events in structural evolution of building composites
}

\author{
Valery Vyrovoy ${ }^{1}$, Oksana Korobko ${ }^{2,}$, and Oleksandr Ielkin $^{3}$ \\ ${ }^{1}$ Odessa State Academy of Civil Engineering and Architecture, Department of Production of Building \\ Products and Constructions, Didrihsona st. 4, 65029 Odessa, Ukraine \\ ${ }^{2}$ Odessa State Academy of Civil Engineering and Architecture, Department of Architectural \\ Structures, Restoration and Reconstruction of Buildings, Constructions and their Complexes, \\ Didrihsona st. 4, 65029 Odessa, Ukraine \\ ${ }^{3}$ Construction company "BUDOVA", Department of Production of Building Products and \\ Constructions, Osipova st. 22, 65000 Odessa, Ukraine
}

\begin{abstract}
The problems of forming the structure of hardening mineral binders as a result of the implementation of basic events and emerging related processes are considered. It is shown that the self-consistency of events and the phenomena caused by them create a dynamic structural portrait of the developing system. Special attention is paid to the fact that changes in temperature and volume deformations are a "shadow" of events and do not allow us to identify the technological event itself. It is pointed out that the development of background processes predetermines the kinetics of self-development of the structures of developing systems
\end{abstract}

\section{Introduction}

Submission of building structures in the form of systems a priori involves their specific structural design. Organization structure depends on the qualitative and quantitative ratios of the starting components, the geometric characteristics of the product and the process conditions and modes of preparation of concrete and its processing into the design system. Thus, the technology is one of the major factors which controlling the structure and thus the properties of composite materials and structures.

In general, the concept of technology (from the Greek techne - art, skill, ability and logos - word, teaching) includes both technology-practice - a set of methods and processes in a particular sector of production and technology, science - scientific description of methods of production and the processes and phenomena that occur in raw material processing to a consumer product. Sakhal D. [1] proposes to use the ideas and methods of system approach for a more complete disclosure of technology concepts. A common feature of the technology, practice, technology, science and technology, the system should be considered as the obligatory presence of a certain set of events, the implementation of which allows obtaining a final product from raw material - in this case, the design system. The events include the chemical, physical, physico-chemical, mechanical, and many other

\footnotetext{
*Corresponding author: okskorobko71@gmail.com
} 
processes and phenomena associated with the interaction of matter and the organization structure of materials. The implementation of each event in the system, as a rule, is accompanied by a change in the energy condition of the interacting substances, which causes variation of temperature and volume of systems.

\section{The change in temperature and volume is a kind of shadow that discards base events}

It has historically developed and methodologically stuck that the phenomena which occur as a result of the passing inevitable basic events are treated as information-rich processes, studying which you can retrospectively describe the event itself. In fact, the change in temperature and volume is a kind of shadow that base events cast. These shadows are organized complexly enough, because there may be possible situations in which the shadow includes a "reflection" of various events of different nature, the intensity of symptoms and time of action. The contours of the shadows, combining and merging with each other, are blurred beyond recognition, which does not allow identifying the events that create them. Plato (428 - 348 BC) in the "Sophist" dialogue noted: "... the shadows, when the darkness is mixed with fire, and then the double reflection, when own light of object and strange ones combine and produce a map that makes sense of contrary to the previous causation visibility." "Mappings" of individual events occurring in a distinctive shadow, are not localized either in time or in space. The causal connection between the events and their accompanying processes are hidden beyond averaged last ones. This leads to a reduction, or even to an involuntary falsification of information about the events taking place, making it difficult to identify effective factors for the technological event control. Especially the informational inadequacy between events and processes initiated by them is typical for highly organized and self-assembled objects-systems. At the same time displayed shadows that occurred as a result of basic events become an active background of themselves which initiates the flow of future events. Not excluded situations in which offset in time of manifestation and deepen the flow of events and the emersion and development of related processes gets reduced, which is typical for systems with multifocal sources of realization of certain events. In these cases it is difficult to separate the events from the processes caused by them. In this connection there arises the problem of the analysis of the possible mechanisms of interaction and mutual influence of the basic events and processes caused by them to initiate the formation of structures with the required parameters.

In the initial stages of research in the study as an object cement-aqueous compositions are taken, which are presented in the form of highly lyophobic coarse dispersion systems with freeze interphase boundary [2, 3]. Isolation of cement-water compositions as independent objects of analysis is due to the indisputable fact of their direct influence on the creation of a multi-purpose concrete material. A distinctive feature of the systems under consideration is a polyiminerality and polydispersity of the particles of dispersed phase, which determines the flow of surface phenomena and related structural transformations of the individual volumes and the entire dispersion system.

The preparation of such compositions is accompanied by a spontaneous re-formation of the interphase interface from S - G into S - L, which leads to a change in the energy state of the system [4]. The average temperature is a faded shade, according to the accepted terminology in the article, of those events that occur in local areas of interphase interactions. Using particles of the same nature, but of different shapes and sizes leads to a temperature change in the local areas of the system. This occurs against occurrence of temperature gradients between the surface and the volume of the particles and between the surface of the particles and the surrounding dispersion medium. Temperature gradients contribute to the development of the internal mass transfer, diffusion processes, activation 
of surface phenomena, and so on. Thus, in a system comprising monomineral particles of the dispersed phase, local disturbances or non-equilibrium states arise spontaneously, which determines further structural reforms.

Polyminerality of the dispersed particles assumes a priori the impact of changes in the temperature of each individual particle on changes in the average temperature of the system. For polymineral and polydisperse systems the following situations are possible: the individual particles are monomineral; - each particle is polymineral formation; - the system is composed of mono- and multimineral particles with different quantitative ratios between them. These cases are typical, with different variations, for real systems and contribute to occurrence of local unbalanced states, the uniqueness of which is hidden in the average temperature changes. It can be concluded that the first peak of heat during the formation of disperse systems is determined by the local exothermic effect which causing local unbalanced conditions. Thereby, the course of further physical and chemical processes of hydration and physical and mechanical processes of structure formation is predetermined. A high concentration of dispersed phase particles suggests that they are located in zones of near or distant coagulation [2]. Under these conditions, all the monoand polymineral particles based considering their polydispersity, are under the influence of unbalanced forces of interparticle interactions [3]. In the lyophobic systems, the reduction of excess surface energy (Gibbs free energy), which converts a system to the nonequilibrium system, happens by reducing the interfacial area of the interface $[4,5]$. The internal reorganization of the system occurs, in which uneven distribution of particles is observed at conservation of their total volume and the total quantity in the system. As a result, the dispersion having fundamentally new structural components - the structural blocks (clusters) and interconnects (inter-cluster) interface [3, 6]. These processes determine the onset and development of subsequent technological events.

The internal distribution of the particles into structural units occurs simultaneously with the lyophilization of their surface. Important and adequately and in detail described in the literature events begin to realize in the system - the physical-chemical processes of hydration of mineral binders $[7,8]$. Implementation of the new developments is carried out on the background of the processes resulting from previous events occurred. Schematic diagram of the development of events and their accompanying processes is shown in Fig. 1.

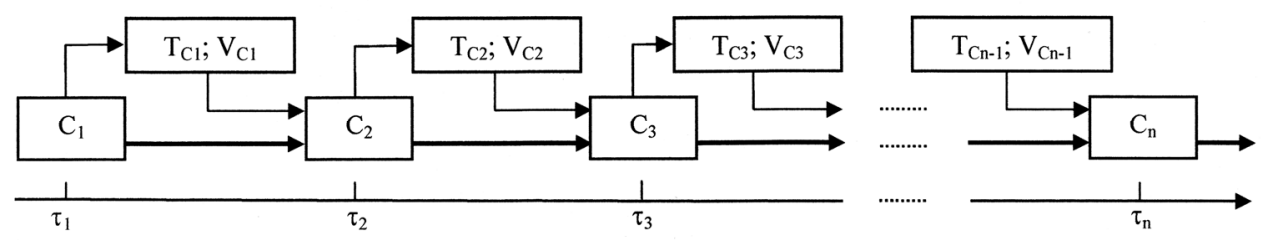

Fig. 1. The scheme of events and their accompanying processes.

$\mathrm{C}_{3} \ldots \mathrm{C}_{\mathrm{n}}$ - development of basic events; $\mathrm{Tc}_{1} \ldots \mathrm{Tc}_{\mathrm{n}-1}$ - temperature change; $\mathrm{Vc}_{1} \ldots \mathrm{Vc}_{\mathrm{n}-1}-$ volume change.

The inevitability of $\mathrm{C}_{2}$ event - in our case the cement mineral grains with water - is obvious. Thus the total flow of kinetics hydration processes is determined by the initial temperature of the interacting phases and temperature difference in local areas of the disperse system. In turn, the resulting related phenomena have an impact on the effectiveness of the implementation of new developments $C_{3} \ldots C_{n}$, which exacerbates the effects of local temperature changes in the overall process of hydration of mineral binders.

Distribution of poly- and mono-mineral grains of cement by structural units involves multifocal nature of the development of hydration of events with a certain time shift, depending on the chemical activity of each individual mineral of cement. In this regard, the accompanying processes caused by events $C_{1}$ and $C_{2}$ are a catalyst for the initiation of $S_{1}$ 
events. In turn, the accompanying processes of events $\mathrm{S}_{2}$ and $\mathrm{C}_{3}$, integrating, can influence on the kinetics of the developments of events $\mathrm{C}_{3}$ and $\mathrm{M}_{1}$, Fig. 2.

It becomes problematic to identify the source of the emergence and development of the processes after a certain period of time. Thus there are a self-support temperature gradients (due to deepening hydration process) between the surface of the particles and their volume, between the surface and the surrounding dispersion medium, and also between the conjugated minerals belonging to the same cement grain [9]. The increased heat release rate of tricalcium aluminate compared to the rate of heat release of tricalcium silicate $[7,10,11]$ suggests an uneven distribution of temperature at polymineral cement grain hydration.

New phase ions directionally migrate as a result of thermal diffusion effects manifestations (Soret effect [12]). This leads to local fluctuations in concentration neoplasms products, forming a complex mosaic of interactions of ions of different species that interact to form nuclei of a new phase and create a kind of amorphous component of the cement matrix in which it is difficult to identify the qualitative and quantitative composition. Using the results of a number of authors $[7,8,13]$ can be offered to discuss thermal and volume kinetic dependence, taking into account the nature of heat and volume changes of individual minerals and of the whole system, Fig. 3.

Experimental data show that the nature of heat polymineral cement grains (Curve 3, Fig 3) does not allow obtaining fully reliable information about events occurring in certain minerals. Even less information contains data on the kinetics of the heat of hydration of cement compositions as a multimineral and polydisperse systems. Multifocal hydration mechanism initiates energy chaos in the system. It is important that the phenomena that occur as a consequence of the events may be initiated for further chemical processes. Systems with a rather complex initial qualitative and quantitative composition of the dispersed phase spontaneously build path of self-initiation of physical and chemical, physical and physical-mechanical processes of hydration and multiscale structural organization. One of the main places in these processes is the thermal effects that arise as a consequence and are the cause of deepening self-organization phenomena. In these time intervals, the structure completes itself with products of a new phase that are produced by the system. Therefore, such systems should be attributed to autopoiesis systems [14].

To identify the factors of self-motion process control system it is important to consider not total energy processes, but its spontaneous striving for minimization of internal energy by forming organized on the principle of long-range or short-range order of the building blocks of different scales, taking into account the local gradients of temperature and concentration. Using the averaged data on temperature change hardening of mineral binders gives little information and does not allow objectively determining effective prescription and technological factors, which allow controlling complex processes of evolution, taking into account the phenomena of self-organization structure of the system.

Exothermic effects, as inevitable processes, arise when certain events are realized and inevitably lead to a change in the volume of the individual particles of the dispersed phase and the entire system. Besides the initial volume change occurs at the moment of the system forming by forming an interfacial boundary surface due to the adsorption of water molecules on the surface of the particles. Volume changes that occur as a result of certain events initiate development of endodeformations. Endodeformations are deformations that occurs "'inside" the system and initiate the emergence and development of physical, chemical and physics-mechanical phenomena and processes of hydration and structure formation of binders and materials based on them. Endodeformations are attendant processes in the implementation of certain events (for example, adsorption processes, physical and chemical processes of hydration, crystallization processes, mass transfer, etc.). To the events that provoke the development of volume changes, it is necessary to attribute 
the arising thermal effects. It can be concluded, that some of the endodeformations as "shadow of shadows" capable themselves to initiate the development of thermal effects.

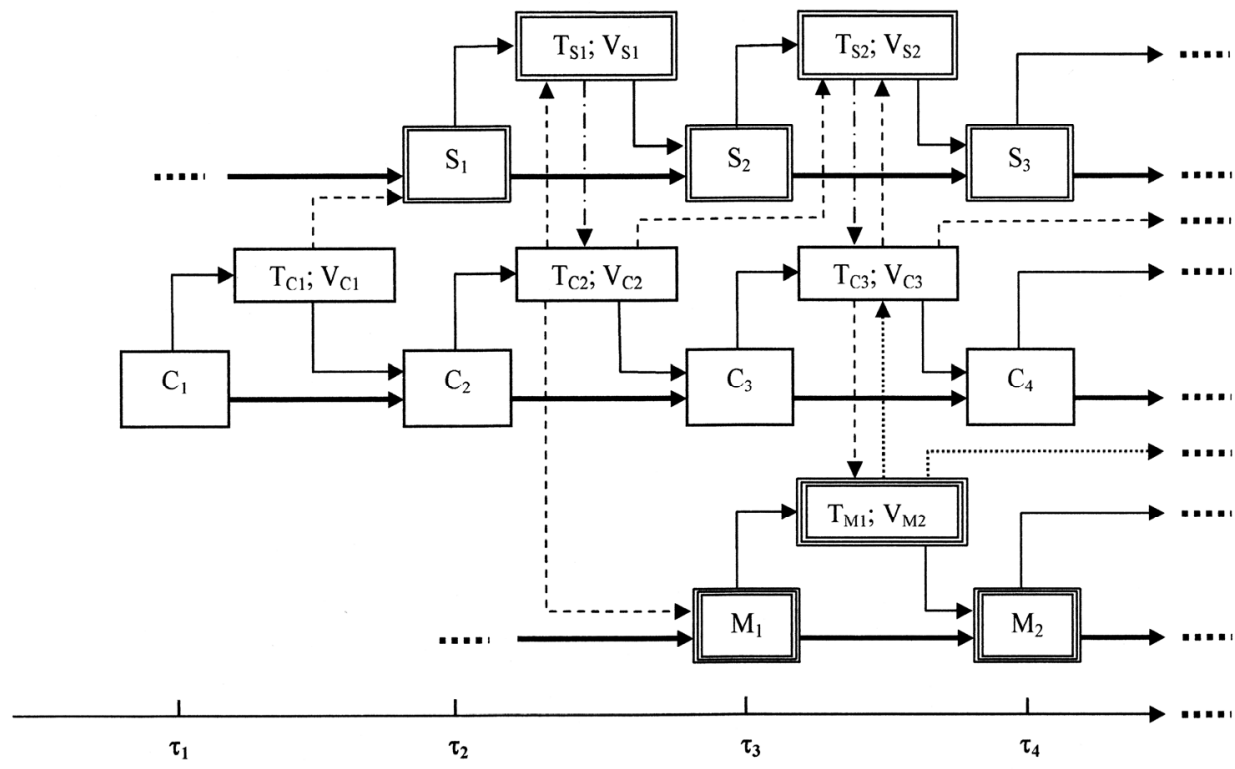

Fig. 2. Development of multifocal interconnectivity of event.

$\mathrm{C}_{3} \ldots \mathrm{C}_{4} ; \mathrm{S}_{1} \ldots \mathrm{S}_{3} ; \mathrm{M}_{1} \ldots \mathrm{M}_{2}$ - basic events; $\mathrm{T}_{\mathrm{c}} ; \mathrm{T}_{\mathrm{s}} ; \mathrm{T}_{\mathrm{M}} ; \mathrm{V}_{\mathrm{c}} ; \mathrm{V}_{\mathrm{s}} ; \mathrm{V}_{\mathrm{M}}$ - the change in temperature and volume at the developments.

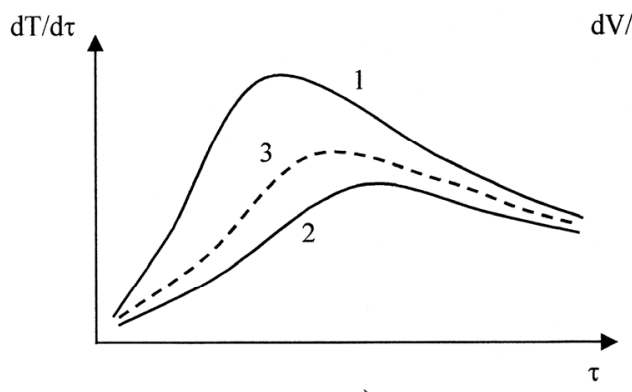

a)

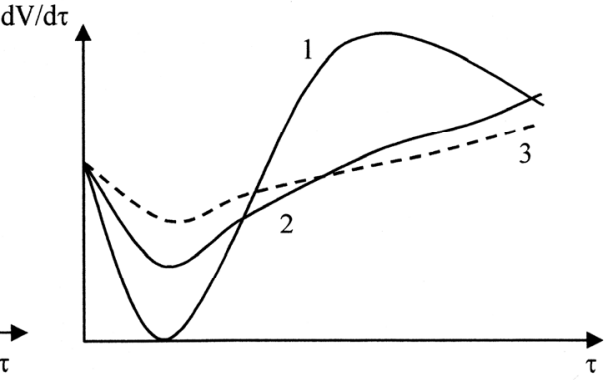

b)

Fig. 3. Kinetics of heat (a) and volume change (b) a single binary and minerals. 1 - tricalcium aluminate $\left(\mathrm{C}_{3} \mathrm{~A}\right) ; 2$ - tricalcium silicate $\left(\mathrm{C}_{3} \mathrm{~S}\right) ; 3$ - average heat polymineral system $\left(\mathrm{C}_{3} \mathrm{~A}+\mathrm{C}_{3} \mathrm{~S}\right)$.

Originating as a result of a complex of diverse processes endodeformation predetermine the path of structural adjustment by creating heat transfer currents that in turn stimulates the further development of physical and chemical processes, causing a new deformation flows. The mutual dependence of thermal and deformation processes can not be considered in isolation from each other. Emerging as a result of events that have already happened, thermal effects and endodeformation define future processes by participating in the organization of multiscale structure of the system. However, their participation is associated with the general process of certain vector of development of the system through the development of local events of structural design of the forming and already formed system. 


\section{Conclusions}

Endodeformation, along with thermal effects, are components of the structural "portrait" of the system and represent it in a move that is its natural state. Dynamics of the structure is based on mutual self-activation and the effect of events caused by them on the processes of each other, a kind of behavior in coordination of material component by forming localization directed brooks and streams. The phenomena of self-organization are realized by mutual stimulation. This can lead to the required macro condition of the system. Technological and methodological efforts are needed, which are able to determine the appropriate factors of behavior management for a class of systems with multifocal sources of structural diversity in order to obtain a final product with a normalized set of properties.

\section{References}

1. D. Sakhal, Technological progress: Concepts, models, assessment (Finance and Statistics, Moscow, 1985)

2. N.B. Uriev, Highly concentrated dispersion systems (Chemistry, Moscow, 1980)

3. V.I. Solomatov, V.N. Vyrovoy, Technological mechanics of concrete (Riga, Latvia, 1985)

4. Y.G. Frolov, Course of Colloid Chemistry (Chemistry, Moscow, 1982)

5. P.A. Rehbinder. Surface phenomena in disperse ( Nauka, Moscow, 1979)

6. V. Solomatov, V. Vyrovoy, V. Dorofeev, A. Sirenko, Composite building materials and constructions of the reduced material capacity (Budivelnik Press, Kiev, 1991)

7. A.A. Pascenco, E.A. Myasnikov, V.S. Vimen, Cement Theory (Budivelnik, Kiev, 1991)

8. J. Stark, B. Wicht, Cement and lime (Oranta, Kiev, 2008)

9. H. Taylor, Cement Chemistry (Mir, Moscow, 1996)

10. A.V. Ursherov-Marshak, Heat release of cement (Research Institute for Scientific and Technical Information and Construction Materials Industry, Moscow, 1980)

11. P.V. Krivenko, R.F. Runova, M.A. Sanitsky, N. I. Rudenko, Alkaline cements (LLC "Osnova", Kiev, 2015)

12. P.P. Kuzmenko, Electro transfer, thermal transfer and diffusion in metals, Vishcha Shkola, Kiev, 1983)

13. O.A. Korobko, V.N. Vyrovoy, collection of scientific works of OSACEA. 3, 113-117 (2001)

14. U. Maturana, F. Varela, The tree of knowledge: the biological roots of human understanding,( "Progress-Tradition" Publishing House, 2001)

15. E. A. Petito, Semiofizika and metaphysical realism. Synergetics. Anthology. 1: 329342. (2013)

16. G., Nicolis, I. Prigogine, Exploring Complexity: An Introduction (LCI, Moscow, 2008)

17. V. Vyrovoy, V. Dorofeev, V. Sukhanov, Composite building materials and constructions. Structure, self-organization, properties (TES Press, Odessa, 2010)

18. S. B. Fic, V. Vyrovoy, V. Dorofeev, The processes of self-organization structure of composite building materials (Politechnika Lublenska Press, Lublin, 2013)

19. V. Vyrovoy, V. Dorofeev, V. Sukhanov, J. Econ. Res. mater., struct., build., constr. 41, 133-139 (2008) 